\title{
TNR ON TOP OF ACCRETING WDS: 2-D SIMULATIONS
}

\author{
S. A. GLASNER ${ }^{1}$, E. LIVNE $^{1}$, J. W. TRURAN ${ }^{2}$ \\ 1. Racah Institute of Physics, The Hebrew University, \\ Jerusalem, Israel \\ 2. University of Chicago, Lab. for Astrophysics and Space \\ Research, Enrico Fermi Institute, Chicago, IL 60637, USA
}

Spherically symmetric models for classical novae as thermonuclear runaways (TNR) of hydrogen-rich, accreted, envelopes on top of cold white dwarfs (WD) are in very good agreement with observations (Starrfield, Sparks \& Truran 1974, 1985; Prialnik, Shara \& Shaviv 1978; Prialnik 1986; MacDonald 1980). Multi-dimensional investigations are needed since the burning rate is very sensitive to the temperature and small fluctuations in temperature can lead to large differences between the flash times of different points on the burning shell; these differences can be much larger than the rise time of the nova light curve. Convection is assumed to be the mechanism that couples the burning process at different points in the shell so that the overall phenomena is spherically symmetric.

We present here 2-D simulations of the accreted envelope for which the only perturbations are those induced by the turbulent nature of the model. Livne (1993) developed an implicit hydro code (VULCAN) which enables such simulation of long term subsonic flows. The initial model consists of a solar mass $\mathrm{C}-\mathrm{O}$ WD at the stage where its luminosity is $L=0.01 \mathrm{~L}_{\odot}$.

Using a 1-D code, we evolve the accreting model in time up to a stage where the temperature at the base of the envelope is already $110^{8} \mathrm{~K}$. We now map the 1-D hydrogen shell on a 2-D grid with identical radial zones. A few underlying $\mathrm{C}-\mathrm{O}$ zones from the 1-D model are also included in the 2-D simulation in order to permit overshoot mixing of $\mathrm{C}-\mathrm{O}$ rich matter by the convective flow into the hydrogen rich accreted envelope. Within a few seconds several convective cells are built spontaneously from the numerical noise. During the first $70 \mathrm{~s}$ nothing violent happens, burning proceeds smoothly while the temperature at the base of the hydrogen shell rises from $10^{8} \mathrm{~K}$ to $1.110^{8} \mathrm{~K}$. Due to the interaction between downward currents and burning, temperature fluctuations of $10-20 \%$ develop within each burning shell. After $70 \mathrm{~s}$ the flow changes its manner and becomes violent. In addition to the intense burning all around the base of the envelope, lo- 


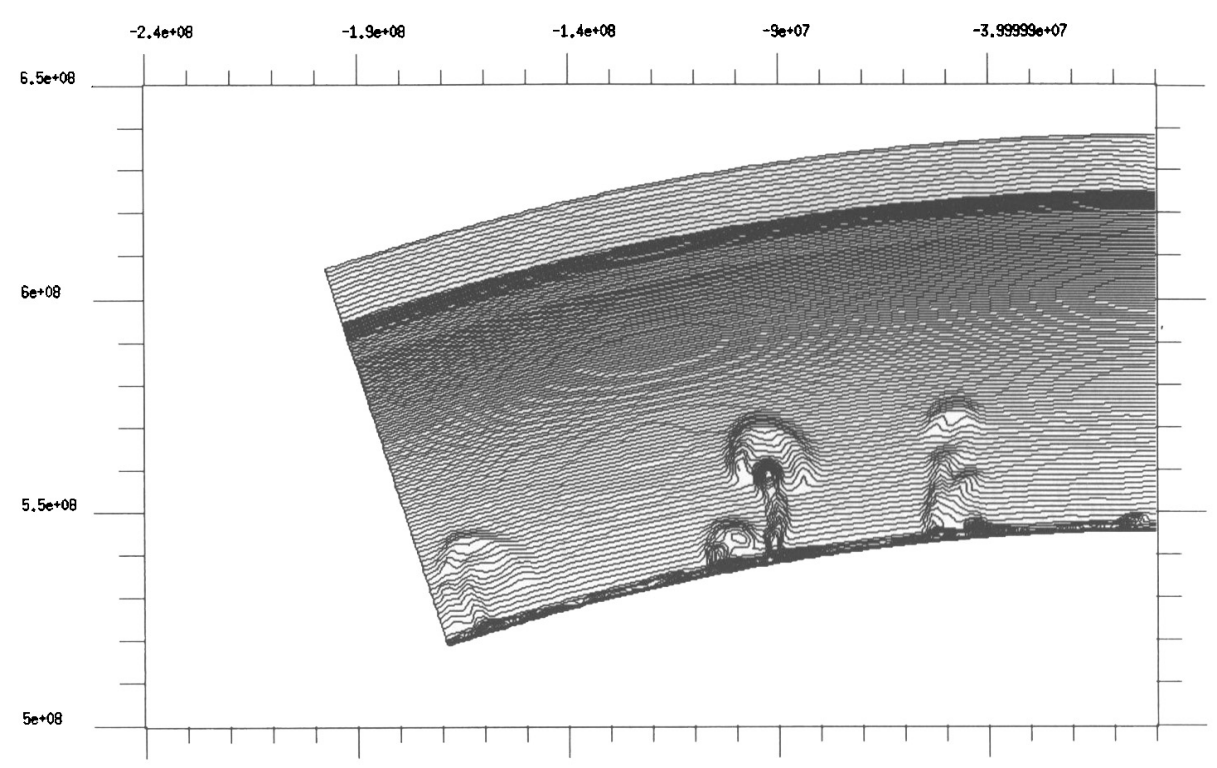

Figure 1. Contours of temperature at time $70 \mathrm{~s}$; axes are cylindrical coordinates $R$ and Z

cal eruptions of very fast burning appear at different points. Convective cells and convective velocities are larger than those predicted by 1-D Mixing Length Theory, convection seems to maintain the burning, rather than regulate it. Since the speed of sound is faster than the matter velocities, pressure waves travel faster than any flow pattern and the pressure quickly equalizes at each spherical shell. Therefore, although the burning process is not spherically symmetric, the overall expansion is almost spherical.

\section{References}

Livne, E., 1993, Ap. J., 412, 634

MacDonald, J., 1980, MNRAS, 191, 933

Prialnik, D., Shara, M.M., Shaviv, G., 1978, A\&A, 62, 339

Prialnik, D., 1986, Ap. J., 310, 222

Starrfield, S., Sparks, W.M., Truran, J.W., 1974, Ap. J. Supp., 28, 247

Starrfield, S., Sparks, W.M., Truran, J.W., 1985, Ap. J., 291, 136 